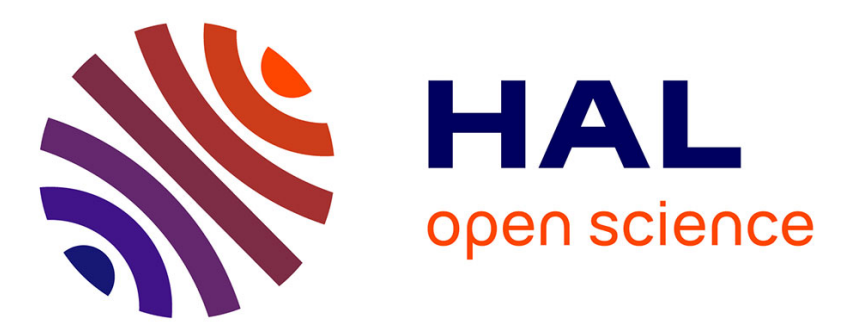

\title{
An efficient and simple refined model for temperature analysis in thin laminated composites
}

Monique Blanc, Maurice Touratier

\section{To cite this version:}

Monique Blanc, Maurice Touratier. An efficient and simple refined model for temperature analysis in thin laminated composites. Composite Structures, 2007, 77 (2), pp.193-205. 10.1016/j.compstruct.2005.07.001 . hal-00101337

\section{HAL Id: hal-00101337 https://hal.science/hal-00101337}

Submitted on 25 Feb 2018

HAL is a multi-disciplinary open access archive for the deposit and dissemination of scientific research documents, whether they are published or not. The documents may come from teaching and research institutions in France or abroad, or from public or private research centers.
L'archive ouverte pluridisciplinaire HAL, est destinée au dépôt et à la diffusion de documents scientifiques de niveau recherche, publiés ou non, émanant des établissements d'enseignement et de recherche français ou étrangers, des laboratoires publics ou privés. 


\title{
An efficient and simple refined model for temperature analysis in thin laminated composites
}

\author{
M. Blanc, M. Touratier* \\ LMSP, UMR 8106 CNRS-ENSAM, Université d’Orléans, 151 Boulevard de l’Hôpital, 75013 Paris, France
}

\begin{abstract}
This paper presents a new refined and simple analytical model for heat conduction problems in multilayered structures based on an equivalent single layer approach, which permits satisfying both continuity conditions for temperature and normal heat flux at interfaces. Continuity conditions at layer interfaces for temperature are automatically assured from the Heaviside step function, while those for normal heat flux allow us to determine the $N-1$ generalized temperatures associated to the Heaviside step function. Finally, using either third order polynomial or trigonometric functions in thickness of the laminate, boundary conditions at the top and bottom of the laminate are used to reduce the number of unknown functions, which is only two. Exact three-dimensional solutions have been calculated here for reference solutions. Numerical results to several problems show the efficacy of the approach on two-dimensional and three-dimensional heat conduction problems in comparison with exact calculations.
\end{abstract}

Keywords: A - layered structures; $\mathrm{B}$-interface, thermal properties; $\mathrm{C}$-laminate theory, thermal analysis

\section{Introduction}

Layered structures are increasingly used in aerospace, automotive vehicles and ships. The so-called advanced composite materials were developed as part of aerospace vehicles during the second half of last century. Nowadays, there are examples of fighter and commercial aircraft, helicopters and gliders whose structures are entirely made of composite materials. Composite constructions are multilayer produced structures, mostly made of flat and curved panels, constituted of several layers or laminae, perfectly bonded together. Other examples of layered structures of interest to the present paper are especially: thermally protected structures in which layers with high thermal properties are used as thermal skins; thermoplastic forming; and, of course,

\footnotetext{
* Corresponding author.

E-mail address: maurice.touratier@paris.ensam.fr (M. Touratier).
}

all laminated structures submitted to heat. Thus, temperature changes often represent a significant factor, and sometimes the predominant cause of failure of composite structures subject to severe environmental loads, see for instance Savoia and Reddy [1].

It has been demonstrated by Padovan $[2,3]$ that the heat conduction problem can be exactly solved for rectangular laminated plates made of orthotropic layers, for any given temperature distribution or thermal conduction conditions prescribed at the top and bottom of the plate. Such a solution has been used for thermoelastic response in laminates [3,1]. The analytical treatment of transient heat conduction for one-dimensional multilayered composites with an automatic solution of the eigenvalue problem is shown in Mikhailov et al. [4]. An exact methodology for analyzing linear, one-dimensional, periodic heating-cooling problems of composites with any number of laminates is given in Han [5]. For composite panels comprising a large number of laminates, the classic method of solution of problems in 
unsteady conduction through layered media which are traditionally based on Green's functions, a transformation technique, or the eigenvalue approach, can be quite forbidding, and even numerical algorithms may be burdensome. Alternatively, continuum models are being developed with the aim of mimicking the thermal responses of composites by using single effective thermal properties (homogenization approach) reflective of the physical makeups of the layered media, see for example the work of Maewal et al. [6].

In laminated composite shells the assumption of linear temperature distribution through the entire shell thickness (in a manner of an equivalent single layer) is hardly adequate, see Bose and Surana [7] and Chattopadhyay et al. [8]. In Carrera [9], and Zhou et al. [10], temperature distribution through the entire plate thickness is quadratic and cubic, respectively, allowing satisfying thermal boundary conditions at the top and bottom. The approach where each lamina of the laminate is modelled using solid finite elements may be highly impractical and may even lead to numerical difficulties as a composite laminate may contain many individual laminas each with a thickness of under $1 \mathrm{~mm}$. These modelling practices and analysis methods either lead to inefficiency or begin to break down in extreme cases where the laminate is either very thin or very thick and containing many laminas. Accurate and efficient heat conduction studies in such applications require simpler geometric models and higher order temperature approximations in all three directions of the element. Finite element approximations for laminated composite applications where the temperature distribution in the shell thickness direction could be of arbitrary polynomial order $p$ have been published by Surana and Orth [11], Surana and Abusaleh [12]. Hierarchical finite element approximations have been proposed for heat conduction analyses by Surana and Orth [13], Bose and Surana [14], while various classic and advanced (for example mixte layerwise or zigzag) theories have been tested in Carrera [15].

In this paper we present a new analytical refined model based on high-order approximation for temperature distribution throughout the thickness of the laminate, which is taken either under a complete polynomial of high-order (till fourth order) or trigonometric expression surimposed to the classic linear distribution. Such a trigonometric form has been introduced in elasticity by Touratier [16], and has been justified in Touratier [17]. This comes from the nature of the exact solutions for laminates which are expressed by means of trigonometric distribution per layer for any physical linear problem, see for example Carslaw and Jaeger [18] for temperature. Then, "generalized temperatures per layer" of order one from the Heaviside step function are surimposed to the throughout thickness temperature field in order to satisfy automatically the continuity con- ditions at layer interfaces for temperature. These generalized temperatures per layer are then determined from the continuity conditions on the normal heat flux at the interfaces. Finally, satisfying boundary conditions on the top and bottom surfaces of the laminates allows to reduce the size of the model used, to only two unknowns. Exact solutions have been derived to verify the accuracy of the proposed model, which gives very good responses on the tested problems.

\section{Formulation of the heat conduction problem in laminates}

A laminated plate occupying the parallelepipedic domain $V$ of lateral edge $\Gamma_{\mathrm{e}}$, of constant thickness $h$ and constituted by $N$ homogeneous anisotropic layers $V^{(i)}$ is considered. The plate is referred to an orthogonal coordinate system $\mathscr{R}=\left\{x_{\alpha}, x_{3}\right\}$, where the $x_{\alpha}(\alpha=1,2)$ axes lie in the reference plane $A$ of the plate and $x_{3}$ is in the transverse direction. The top and bottom faces of the plate and the layer interfaces, located at $x_{3}=z=z_{\mathrm{t}}, z_{\mathrm{b}}$ and $z_{i+1}(i=1, N-1)$, are denoted by $\Gamma_{\mathrm{t}}, \Gamma_{\mathrm{b}}$ and $\Gamma_{i}$, respectively. The temperature field in the laminate $V$ is denoted by $T\left(x_{1}, x_{2}, x_{3}, \tau\right)$, and the heat flux components by $q_{m}\left(x_{1}, x_{2}, x_{3}, \tau\right)$, in respect to the above coordinate system $\mathscr{R}$, where $\tau$ is the time. It is assumed that the layers have a orthotropic symmetry. Consequently, thermal conductivities $k_{\alpha \beta}(\alpha \neq \beta)$ are zero all along in this paper. Therefore, the heat conduction is governed in the laminate $V$ by the following boundary-value problem for any time $\tau>0\left(T_{, \alpha \beta}\right.$ stands with the derivative of $T$ in respect to $x_{\alpha}$ and $x_{\beta}$, and similarly for $T_{, \tau}$ and $T_{, 3}$ ):

$$
\begin{aligned}
& \rho C_{v} T_{, \mathrm{t}}=k_{i i} T_{, i i}+\rho w, \quad \text { in } V \\
& \pm k_{33} T_{, 3}=\bar{q}_{3} \text { or } T=\bar{T}^{\mathrm{b}, \mathrm{t}} \text { at bottom } \Gamma_{\mathrm{b}} \text {, top } \Gamma_{\mathrm{t}} \\
& {\left[-k_{33} T_{, 3}\right]=0 \text { and }[T]=R\left(-k_{33} T_{, 3}\right) / A \text { at } \Gamma_{i}} \\
& Q=-q_{\alpha} n_{\alpha} \text { or } T=\bar{T}^{\mathrm{e}} \text { on } \Gamma_{\mathrm{e}} \\
& T\left(x_{1}, x_{2}, x_{3}, 0\right)=T_{0}\left(x_{1}, x_{2}, x_{3}\right) \text { at time } \tau=0
\end{aligned}
$$

where $k_{i i}$ (no summation on $i$ ) are constant thermal conductivities, $\bar{T}, \bar{Q}$ and $\bar{q}_{m}$ are prescribed temperatures, heat quantities and heat fluxes, respectively, $\rho$ is the mass density, $C_{v}$ is the specific heat at constant volume, and the bracket [.] denotes the jump in the enclosed argument, $n_{\alpha}$ are the in plane components of the unit normal outward to the edge $\Gamma_{\mathrm{e}}$. Note that $\rho w$ is an internal source of heat and in Eq. (3), $R$ is a thermal resistance and $A$ the contact area. Moreover, unless otherwise stated, throughout the paper greek and italic subscripts assume the values $(1,2)$ and $(1,2,3)$, respectively, and the summation convention over repeated indices is used. Due to the lamination of the plate, all material properties in the above equations are of course dependant on those of each layer. Solving Eqs. (1)-(5) 
exactly give us the exact three-dimensional solution of the expected thermal conduction problem. Examples will be given below for several laminated plates, used to validate the new model presented in this paper.

Finally, an approximated solution of the above threedimensional problem is deduced in a classic manner either by analytical or computational approaches (finite elements for example), here from a variational form deduced from an integration performed throughout the thickness of the above equations, as:

$$
\begin{aligned}
\forall \Theta & \in C^{1}([0, h], \mathbb{R}), \forall \tau, \forall\left(x_{1}, x_{2}\right): \\
& \times \int_{z=0}^{h} \rho C_{V} T_{, \mathrm{t}} \Theta \mathrm{d} z=\int_{z=0}^{h}-q_{3} \Theta^{\prime} \mathrm{d} z \\
& +\int_{z=0}^{h} k_{\alpha \alpha} T_{, \alpha \alpha} \Theta \mathrm{d} z+\int_{z=0}^{h} \rho w \Theta \mathrm{d} z+\left[q_{3} \cdot \Theta\right]_{\Gamma_{\mathrm{b}}}^{\Gamma_{\mathrm{b}}}
\end{aligned}
$$

We can choose $\Theta$ given by:

$\Theta(0)=0$ if $T$ is prescribed on $\Gamma_{\mathrm{b}}$ and $\Theta(h)=0$ if $T$ is prescribed on $\Gamma_{\mathrm{t}}$.

$$
\begin{gathered}
\forall \Theta \in C^{1}([0, h], \mathbb{R}),\left\{\begin{array}{l}
\Theta(0)=0 \quad \text { if } T \text { is prescribed on } \Gamma_{\mathrm{b}} \\
\Theta(h)=0 \text { if } T \text { is prescribed on } \Gamma_{\mathrm{t}}
\end{array},\right. \\
\forall \tau, \forall\left(x_{1}, x_{2}\right): \int_{z=0}^{h} \rho C_{V} T_{, \mathrm{t}} \Theta \mathrm{d} z=\int_{z=0}^{h}-q_{3} \Theta^{\prime} \mathrm{d} z \\
+\int_{z=0}^{h} k_{\alpha \alpha} T_{, \alpha \alpha} \Theta \mathrm{d} z+\int_{z=0}^{h} \rho w \Theta \mathrm{d} z+\left[Q_{\Gamma} \cdot \Theta_{\mid \Gamma}\right]
\end{gathered}
$$

where $Q_{\Gamma}$ is the heat quantity prescribed (natural boundary conditions) on the part $\Gamma_{Q}$ of the boundary surface $\Gamma_{\mathrm{bt}}=\Gamma_{\mathrm{t}} \cup \Gamma_{\mathrm{b}}$ of the volume $V$ of the laminate.

Finally, $\Gamma_{T}$ is the complementary part of $\Gamma_{Q}$ in $\Gamma_{\mathrm{bt}}$, where temperatures are prescribed (essential boundary conditions).

\section{The equivalent single layer model}

The laminate being subdivided into $N$ discrete layers, see Fig. 1, the temperature field is then assumed in the following form:

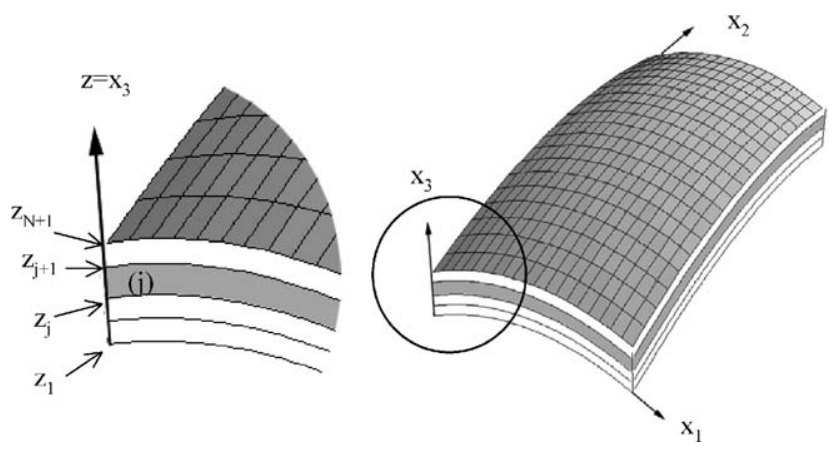

Fig. 1. The composite laminate. The index in parentheses indicates the layer $V^{(i)}$.

$$
\begin{aligned}
& T\left(x_{1}, x_{2}, z, \tau\right)= P(z)+\sum_{i=1}^{N-1}\left(\Delta^{(i, i+1)}+\left(z-z_{i+1}\right) \Lambda^{(i, i+1)}\right) \\
& \times H\left(z-z_{i+1}\right) \quad \text { with } \\
& P(z)= T_{0}\left(x_{1}, x_{2}, \tau\right)+\left(z-\frac{h}{2}\right) T_{1}\left(x_{1}, x_{2}, \tau\right) \\
&+\sum_{j=2}^{\mathrm{nf}+1} F_{j}(z) T_{j}\left(x_{1}, x_{2}, \tau\right)
\end{aligned}
$$

where $z=x_{3}$, and $\mathrm{nf}$ is the number of surimposed functions, such as:

$$
\left\{\begin{array}{l}
F_{2}(z)=\left(z-\frac{h}{2}\right)^{2} \\
F_{3}(z)=\left(z-\frac{h}{2}\right)^{3} \\
F_{4}(z)=\left(z-\frac{h}{2}\right)^{4}
\end{array}\right.
$$

and

$$
\left\{\begin{array}{l}
F_{2}(z)=\sin \left(\frac{\pi z}{h}\right) \\
F_{3}(z)=\cos \left(\frac{\pi z}{h}\right)
\end{array}\right.
$$

(as suggested for example in Ossadzow-David and Touratier [19], for the expression in which a trigonometric form is surimposed to the classic linear distribution.)

In Eq. (7), $H$ is the Heaviside step function defined by:

$H(u)=1$ if $u \geqslant 0$ and $H(u)=0$ if $u<0$

while $\Delta^{(i, i+1)}$ are introduced to incorporate a possible thermal resistance at layer interfaces, and $\Lambda^{(i, i+1)}$ are the generalized temperatures per layer which permit with the help of the Heaviside step function to exactly satisfy the interface continuity temperature at the layer interfaces. Both $\Delta^{(i, i+1)}$ and $\Lambda^{(i, i+1)}$ are functions on $x_{1}$, $x_{2}, \tau$. The generalized temperatures per layer are then determined from the continuity conditions on the normal component of the heat flux at the interfaces. Therefore, from Eq. (3), the continuity condition at the interface $\Gamma_{i}\left(z=z_{i+1}\right)$ for the normal heat flux is expressed by:

$$
\left\{\begin{array}{l}
\left.\left.k_{33}^{(i)} \quad P^{\prime}\left(z_{i+1}\right)+\sum_{j=1}^{i-1} \Lambda^{(j, j+1)}\right)=k_{33}^{(i+1)} P^{\prime}\left(z_{i+1}\right)+\sum_{j=1}^{i} \Lambda^{(j, j+1)}\right) \\
\Delta^{(i, i+1)}=\frac{R^{(i)}}{A^{(i)}} k_{33}^{(i)} \quad P^{\prime}\left(z_{i+1}\right)+\sum_{j=1}^{i-1} \Lambda^{(j, j+1)}
\end{array}\right.
$$

where $R^{(i)}$ is the thermal resistance, $P^{\prime}$ is the first derivative of $P$ ( see Eq. (7)) in respect to $z, A^{(i)}$ is the contact area at the interface $z=z_{i+1} ; k_{33}^{(i)}$ and $k_{33}^{(i+1)}$ the thermal conductivities, respectively in the layers $i$ and $i+1$. From the following Eq. (12), the $2(N-1)$ functions $\Lambda^{(i, i+1)}$ and $\Delta^{(i, i+1)}$ in Eq. (11), can then be expressed in terms of thermal conductivities, and the first derivative of $P(z)$ as: 


$$
\begin{aligned}
& \left.\Lambda^{(i, i+1)}=\sum_{j=2}^{i+1} \prod_{m=j}^{i+1} \frac{k_{33}^{(m-1)}-k_{33}^{(m)}}{k_{33}^{(m)}}\right) P^{\prime}\left(z_{j}\right) \quad \text { and } \\
& \Delta^{(i, i+1)}=\frac{R^{(i)}}{A^{(i)}} \frac{k_{33}^{(i)} k_{33}^{(i+1)}}{k_{33}^{(i)}-k_{33}^{(i+1)}} \Lambda^{(i, i+1)}
\end{aligned}
$$

Moreover, the thermal boundary conditions at the top and bottom of the laminate given by Eq. (2) allows one to eliminate two throughout the thickness generalized temperatures in $P(z)$. We choose to eliminate the generalized temperatures as follows:

(i) Using $P(z)$ given by the polynomial expression (7), if a fourth order approximation is desired as in Eq. (8), $T_{4}\left(x_{1}, x_{2}, \tau\right)$ and $T_{3}\left(x_{1}, x_{2}, \tau\right)$ can be eliminated in function of $T_{0}\left(x_{1}, x_{2}, \tau\right), T_{1}\left(x_{1}, x_{2}, \tau\right)$ and $T_{2}\left(x_{1}, x_{2}, \tau\right)$; while, if a third order approximation is required, then $T_{3}\left(x_{1}, x_{2}, \tau\right)$ and $T_{2}\left(x_{1}, x_{2}, \tau\right)$ can be expressed in terms of $T_{0}\left(x_{1}, x_{2}, \tau\right)$ and $T_{1}\left(x_{1}, x_{2}, \tau\right)$; finally, if a second order approximation is needed, $T_{2}\left(x_{1}, x_{2}, \tau\right)$ and $T_{1}\left(x_{1}, x_{2}, \tau\right)$ can be eliminated in function of the average temperature $T_{0}\left(x_{1}, x_{2}, \tau\right)$ at the mid-plane of the laminate.

(ii) Using $P(z)$ from Eq. (10) given by the trigonometric form (Eq. (9)), surimposed to the classic linear approximation, we can take into account of a higher-order throughout the thickness approximation in which $T_{2}\left(x_{1}, x_{2}, \tau\right)$ and $T_{3}\left(x_{1}, x_{2}, \tau\right)$ can be expressed in terms of $T_{0}\left(x_{1}, x_{2}, \tau\right)$ and $T_{1}\left(x_{1}\right.$, $\left.x_{2}, \tau\right)$.

(iii) $\mathrm{We}$ designate $\mathrm{ESL} 4 \mathrm{C}$ the numerical model obtained by using functions as in Eq. (7) with Heaviside step functions in each layer and by ESL_4 without Heaviside step functions. In the same way, ESL_SC_C designates the numerical model obtained by using functions as in Eq. (9) with Heaviside step functions in each layer and ESL_SC without Heaviside step functions. More generally we can designate by ESL $\_r$ the numerical model obtained by using polynomial function of degree $r$ without Heaviside step functions, and by ESL_r $\mathrm{C}$ the numerical model deduced from a polynomial function of degree $r$ incorporating Heaviside step functions.

As result, Eq. (7) with Eqs. (8), (9) and (12) into Eq. (2) involves various temperature fields depending on the kind of the boundary conditions, and the temperature approximation kept. Then, the final temperature field for all the above approximations can be written as:

$$
T\left(x_{1}, x_{2}, z, \tau\right)=\sum_{j=1}^{\mathrm{nu}} \theta_{j}(z) \cdot \tilde{T}_{j}\left(x_{1}, x_{2}, \tau\right)+\Xi\left(x_{1}, x_{2}, \tau\right)
$$

where $\theta_{j}(z), j \in\{1, \ldots, \mathrm{nu}\}$ are depending on $P(z)$ and $P^{\prime}(z)$, and $\Xi\left(x_{1}, x_{2}, \tau\right)$ is a function of the thermal loading conditions, thermal conductivities and thermal resistances; $\mathrm{nu}=\mathrm{nf}$ is the number of unknown functions.

The thermal boundary value problem is solved by introducing the temperature field given by Eq. (13) into the variational form through the thickness, given by Eq. (6b), requiring to solve: $\forall m \in\{1, \mathrm{nu}\}$

$$
\begin{aligned}
\sum_{j=1}^{\mathrm{nu}} & \int_{z=0}^{h} \rho C_{V}\left(\theta_{j} \tilde{T}_{j, \tau}+\Xi_{, \tau}\right) \cdot \theta_{m} \mathrm{~d} z \\
= & \sum_{j=1}^{\mathrm{nu}}\left(\int_{z=0}^{h}-k_{33} \tilde{T}_{j} \theta_{j}^{\prime} \theta_{m}^{\prime} \mathrm{d} z+\int_{z=0}^{h} k_{\alpha \alpha}\left(\tilde{T}_{j \alpha \alpha} \theta_{j}+\Xi_{, \alpha \alpha}\right) \theta_{m} \mathrm{~d} z\right) \\
& +\int_{z=0}^{h} \rho w \theta_{m} \mathrm{~d} z+\left[Q_{\Gamma_{\mathrm{bt}}} \cdot \theta_{m \mid \Gamma_{\mathrm{bt}}}\right]
\end{aligned}
$$

to compute the unknown functions $\tilde{T}_{j}(z), j \in\{1, \ldots$, $\mathrm{nu}\}$.

It is easy to derive Eq. (14) under the following compact form, as

$$
\begin{aligned}
\forall m & \in\{1, \mathrm{nu}\}, \quad \sum_{j=1}^{\mathrm{nu}}\left(c_{j m} \frac{\partial \tilde{T}_{j}}{\partial \tau}+b_{j m} \tilde{T}_{j}+a_{\alpha j m} \tilde{T}_{j, \alpha \alpha}\right)-\Phi_{m} \\
& =0
\end{aligned}
$$

with

$$
\begin{aligned}
c_{j m}= & \int_{z=0}^{h} \rho C_{V} \theta_{j} \cdot \theta_{m} \mathrm{~d} z, \quad b_{j m}=\int_{z=0}^{h} k_{33} \cdot \theta_{j}^{\prime} \cdot \theta_{m}^{\prime} \mathrm{d} z, \\
a_{\alpha j m}= & -\int_{z=0}^{h} k_{\alpha \alpha} \cdot \theta_{j} \cdot \theta_{m} \mathrm{~d} z \\
\Phi_{m}= & -\int_{z=0}^{h} \rho C_{V} \Xi_{, \tau} \cdot \theta_{m} \mathrm{~d} z+\int_{z=0}^{h} k_{\alpha \alpha} \Xi_{, \alpha \alpha} \cdot \theta_{m} \mathrm{~d} z \\
& +\int_{z=0}^{h} \rho w \theta_{m} \mathrm{~d} z+\left[Q_{\Gamma_{\mathrm{bt}}} \cdot \theta_{m \mid \Gamma_{\mathrm{bt}}}\right]
\end{aligned}
$$

In the next section we choose several examples such that this system of $N$ partial differential equations has an exact analytical solution. So we will be able to compare the approximation given by numerical methods with the exact solution.

In general case we can use classical methods of resolution as finite differences or Fourier's decomposition to solve the reduced problem given by Eq. (15).

\section{Numerical evaluations of the new refined model}

In every example we use dashed line to represent the exact solution. All numerical results are derived from the Mathematica system [20]. 
4.1. Computations of the heat conduction in an infinite sandwich media

According to Fig. 2, we consider an infinite sandwich media, composed by three different isotropic layers of constant thickness, under a thermal loading. In this example we use a thermal loading in the thickness of the media and dimensionless quantities.

For these data the exact solution is given by:

Layer 1

$\left(1.42857 T t+4.95635 \times 10^{-18} W+0\right) z+\frac{W \sin (\pi z)}{\pi^{2}}+0$

Layer 2

$$
\begin{aligned}
2.47818 \times 10^{-18} z W= & \frac{\sin (\pi z) W}{2 \pi^{2}}+0.0297775 W \\
& +\operatorname{Tt}(0.714286 z+0.142857)
\end{aligned}
$$

Layer 3

$$
\begin{gathered}
4.95635 \times 10^{-18} z W+\frac{\sin (\pi z) W}{\pi^{2}}-4.95635 \times 10^{-18} W \\
+0 \cdot z+T t(1.42857 z-0.428571)
\end{gathered}
$$

The ESL_3_C (cf. Eq. (8)) approximation gives:
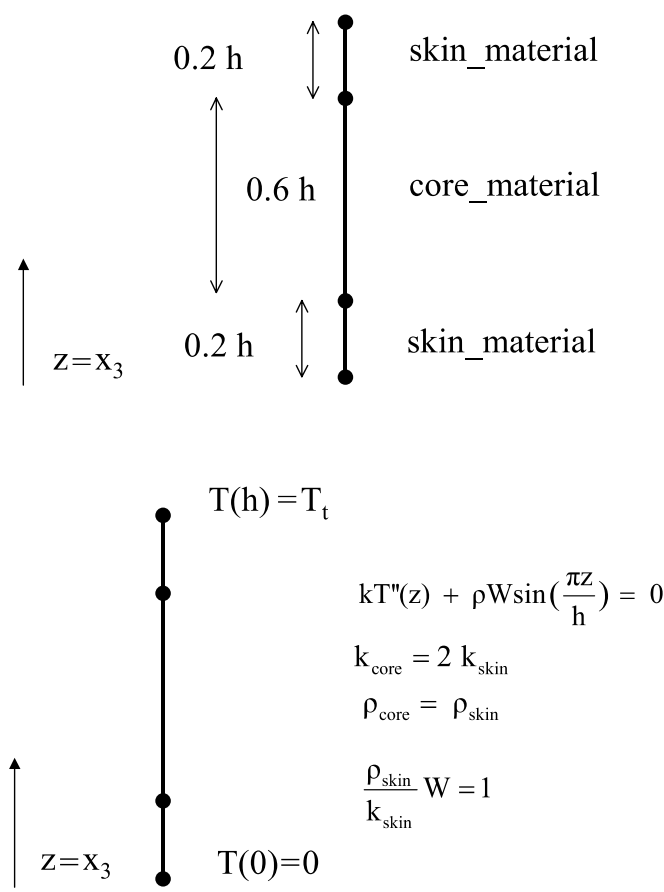

Fig. 2. Geometric and thermal properties, and thermal boundary conditions for the infinite sandwich media.
Layer 1

$$
\begin{aligned}
(- & \left.1.16119 \times 10^{-14} T t-1.17174 \times 10^{-15} W\right) z^{3}+(2.1826 \\
& \left.\times 10^{-14} T t-0.214792 W\right) z^{2}+(1.42857 T t \\
& +0.343667 W) z+0 \cdot T t+0 \cdot W
\end{aligned}
$$

Layer 2

$$
\begin{aligned}
& \left(-1.16119 \times 10^{-14} T t-1.17174 \times 10^{-15} \mathrm{~W}\right) \mathrm{z}^{3}+(2.1826 \\
& \left.\quad \times 10^{-14} \mathrm{Tt}-0.214792 \mathrm{~W}\right) \mathrm{z}^{2}+(0.714286 T t \\
& \quad+0.214792 \mathrm{~W}) \mathrm{z}+0.142857 \mathrm{Tt}+0.025775 \mathrm{~W}
\end{aligned}
$$

Layer 3

$$
\begin{aligned}
& \left(-1.16119 \times 10^{-14} T t-1.17174 \times 10^{-15} \mathrm{~W}\right) z^{3}+(2.1826 \\
& \left.\quad \times 10^{-14} T t-0.214792 \mathrm{~W}\right) z^{2}+(1.42857 T t \\
& \quad+0.0859167 \mathrm{~W}) z-0.428571 T t+0.128875 \mathrm{~W}
\end{aligned}
$$

The ESL_SC_C (cf. Eq. (8)) approximation gives:

Layer 1

$0.136366 z W+3.24858 \times 10^{-17} \cos (\pi z) W+0.0536537$

$\times \sin (\pi z) W-3.24858 \times 10^{-17} \mathrm{~W}+1.42857 \mathrm{Ttz}$

Layer 2

$0.714286 z T t-0.1428571 T t+0.0272733 W+2.77556$

$\times 10^{-17} \mathrm{Wz}+3.24858 \times 10^{-17} \mathrm{~W} \cos (\pi z)+0.0536537 \mathrm{~W}$

$\times \sin (\pi z)$

Layer 3

$$
\begin{array}{r}
1.42857 z T t-0.42857 T t+0.136366 W-0.136366 W z \\
+3.24858 \times 10^{-7} W \cos (\pi z)+0.0536537 W \sin (\pi z)
\end{array}
$$

In Fig. 3, we compare the numerical results with DL (discrete layer) and CDL (constrained discrete layer) approximation using one discrete layer in each layer cf. [21].

In Fig. 3a, we compare the numerical results without continuity of flux (ESL_r, ESL_SC and DL).

In Fig. 3b, we compare the numerical results with continuity of flux (ESL_r_C, ESL_SC_C and CDL).

We can observe the benefit obtained by introducing the continuity of the flux at interfaces with ESL models.

In Table 1, we give the number of unknown functions (cf. Eq. (15)) for each method.

Table 1

Number of unknown functions used in models of Fig. $3 \mathrm{a}$ and $\mathrm{b}$

\begin{tabular}{lllllll}
\hline & ESL_2 & ESL_3 & ESL_4 & ESL_SC & DL & CDL \\
\hline $\mathrm{nu}$ & 1 & 2 & 3 & 2 & 5 & 3
\end{tabular}



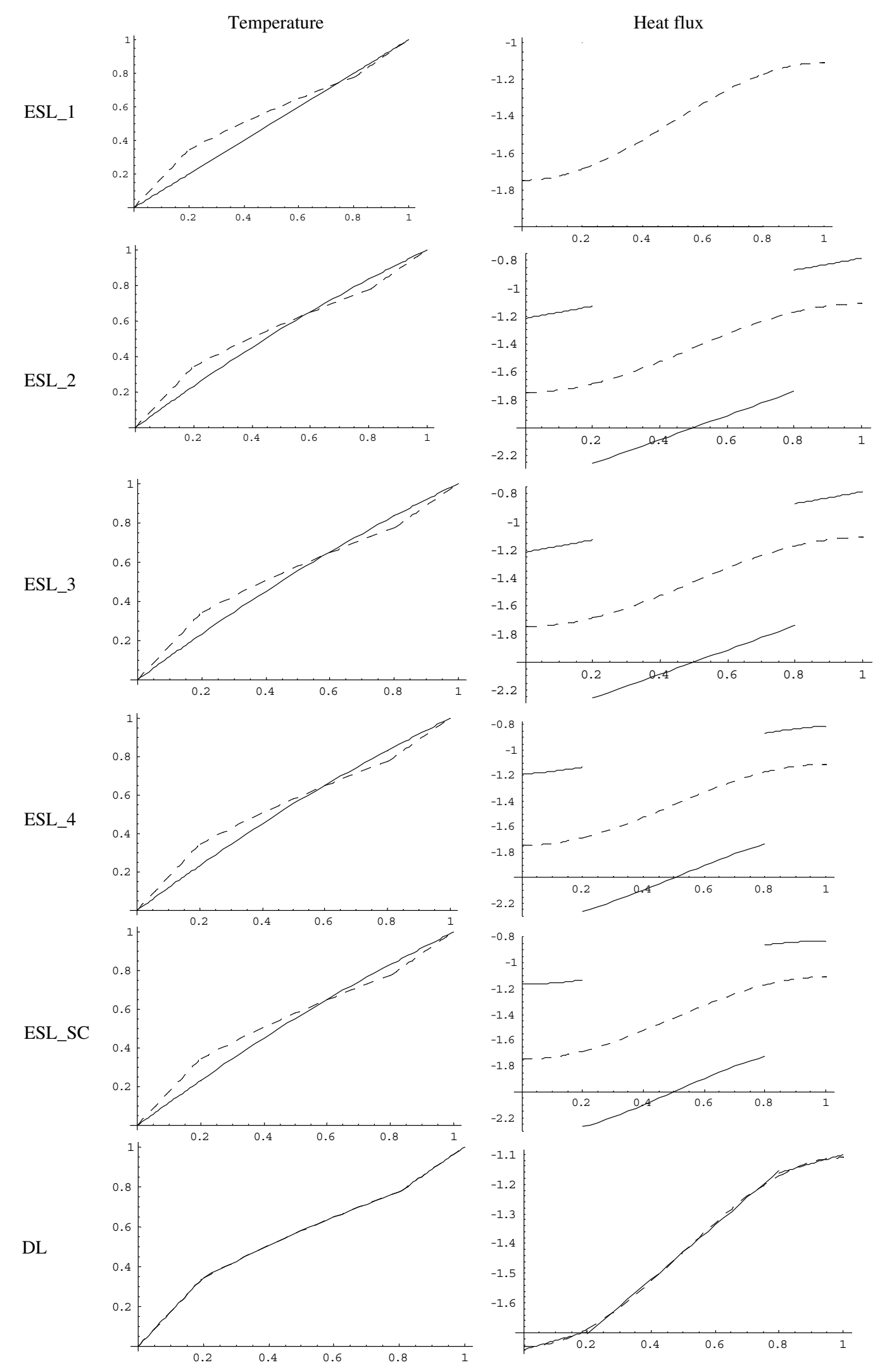

Fig. 3. Infinite sandwich media: temperature and heat flux distributions (a) without continuity of flux at the interfaces, (b) taking into account of the interface continuity for the heat flux. Exact solution in dashed lines. 

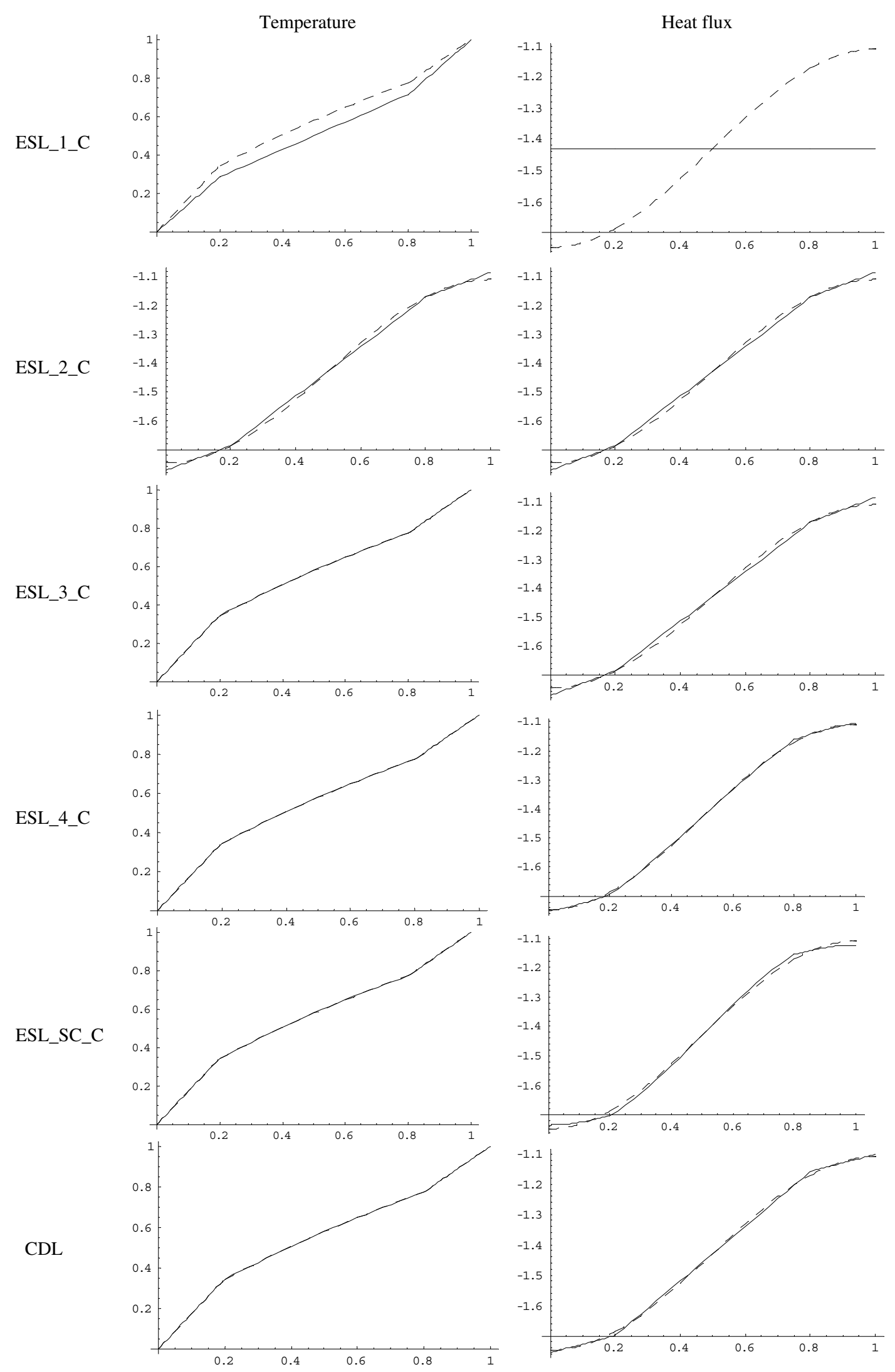

Fig. 3 (continued) 


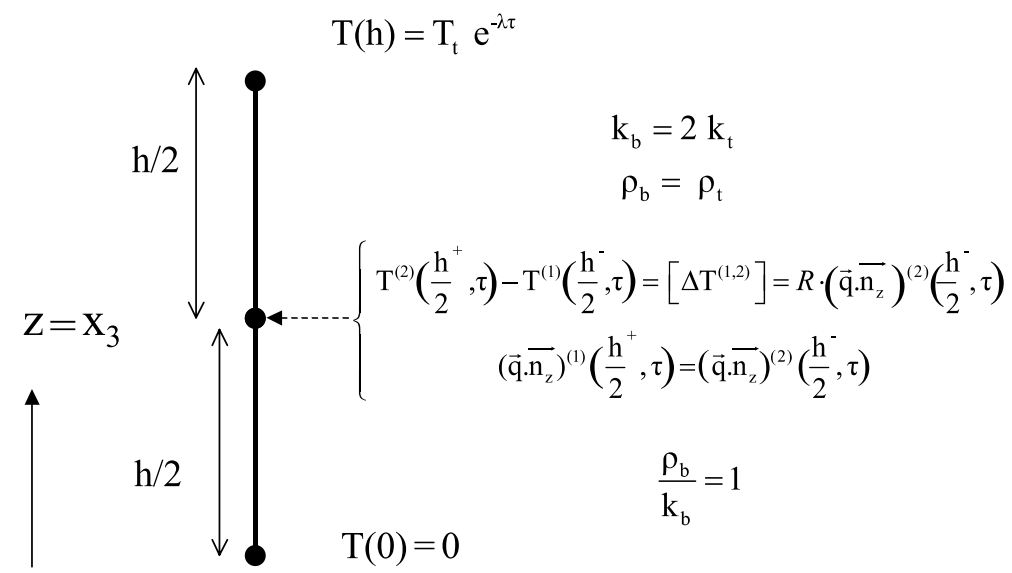

Fig. 4. Data of the heat conduction problem in an infinite bi-layer media incorporating a thermal resistance.
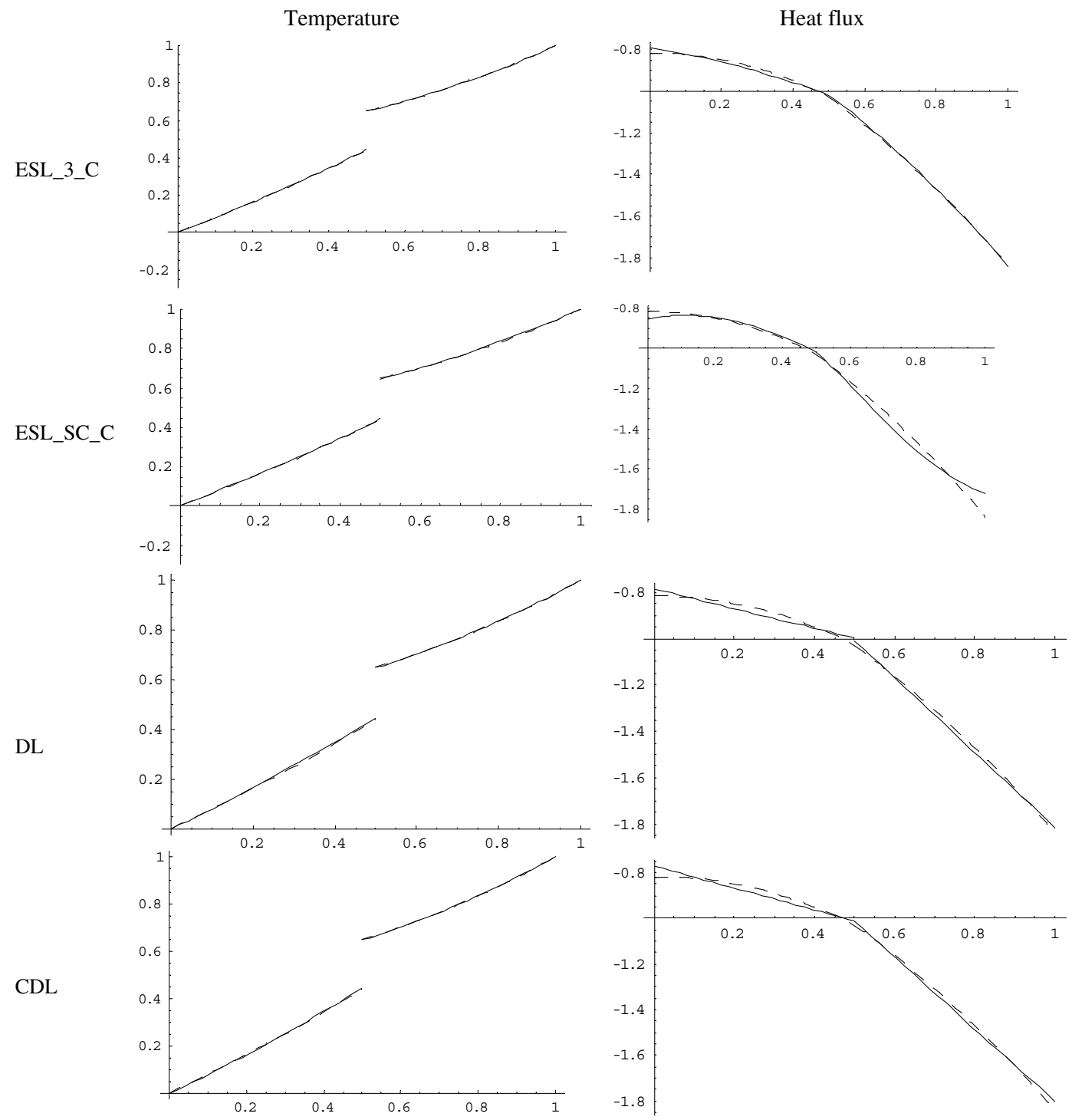

Fig. 5. Distribution of the temperature and heat flux for an infinite bi-layer media
CDL models are compared to the exact solution. Exact solution in dashed lines. 
Geometric and thermal properties of the sandwich

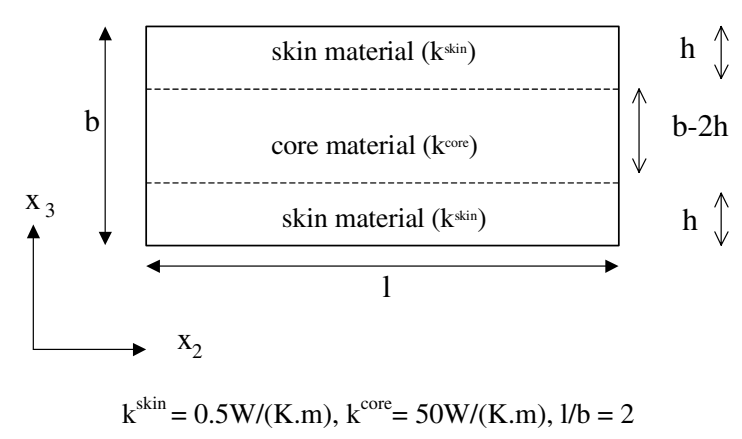

Boundary conditions prescribed at top, bottom and lateral edges of the sandwich

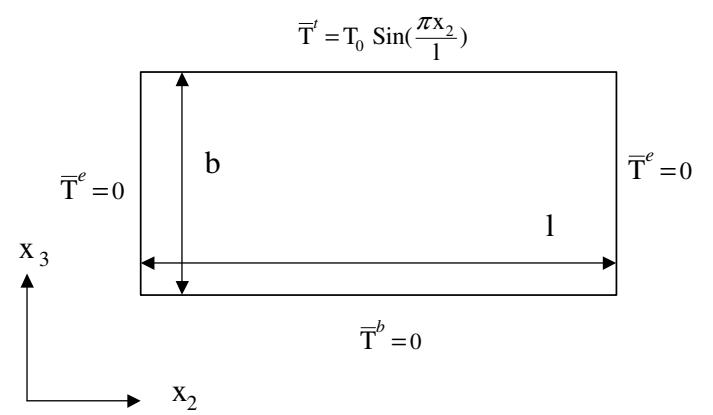

Fig. 6. Geometric and thermal properties, and thermal boundary conditions for the two-dimensional sandwich problem.

Remark. According to Ref. [21], it is important to note that DL and CDL models are derived from a discrete layer approach along the thickness of the laminate, in the manner of a three-dimensional finite element discretisation in thickness direction, in each layer.

\subsection{Computations of the heat conduction in an infinite bi-} layer media with contact resistance and periodic heat cooling load

We consider an infinite sandwich media, according to Fig. 4, composed by two distinct isotropic layers of con-

Table 2

Distribution of the temperature $T / T_{0}\left(x_{2}=1 / 2, z\right)$ along the thickness at the station $x_{2}=1 / 2$, for the composite sandwich shown in Fig. 6

\begin{tabular}{llll}
\hline$z / h$ & Exact & ESL_3_C & ESL_SC_C \\
\hline 0 & 0.0000 & 0.0000 & 0.0000 \\
0.1 & 0.0130 & 0.0130 & 0.0131 \\
$0.2-$ & 0.0264 & 0.0265 & 0.0265 \\
$0.2+$ & 0.0264 & 0.0265 & 0.0265 \\
0.3 & 0.0269 & 0.0269 & 0.0269 \\
0.4 & 0.0280 & 0.0280 & 0.0280 \\
0.5 & 0.0298 & 0.0297 & 0.0297 \\
0.6 & 0.0324 & 0.0323 & 0.0323 \\
0.7 & 0.0357 & 0.0357 & 0.0357 \\
$0.8-$ & 0.0399 & 0.0400 & 0.0400 \\
$0.8+$ & 0.0399 & 0.0400 & 0.0400 \\
0.9 & 0.5136 & 0.5195 & 0.5196 \\
1 & 1.0000 & 1.0000 & 1.0000 \\
\hline
\end{tabular}

stant thickness with a thermal loading. In this example we use a periodic heating-cooling thermal loading (see Fig. 4).

In the numerical application we use $h=1, \lambda=2$, $R=.2$.

For these numerical values the exact solution is given by:

Layer 1

$0.5773597 t \sinh (\sqrt{2} z)$

Table 3

Distribution of the component $q_{2} b /\left(k^{\text {skin }}\right)\left(x_{2}=1, z\right)$ of the heat flux along the thickness at the station $x_{2}=1$, for the composite sandwich shown in Fig. 6

\begin{tabular}{llll}
\hline$z / h$ & Exact & ESL_3_C & ESL_SC_C \\
\hline 0 & 0 & 0 & 0.0000 \\
0.1 & 0.0102 & 0.0102 & 0.0103 \\
$0.2-$ & 0.0207 & 0.0208 & 0.0208 \\
$0.2+$ & 2.0725 & 2.0793 & 2.0819 \\
0.3 & 2.1088 & 2.1131 & 2.1142 \\
0.4 & 2.1973 & 2.1968 & 2.1955 \\
0.5 & 2.3402 & 2.3359 & 2.3334 \\
0.6 & 2.5409 & 2.5359 & 2.5345 \\
0.7 & 2.8044 & 2.8022 & 2.8036 \\
$0.8-$ & 3.1372 & 3.1406 & 3.1437 \\
$0.8+$ & 0.0314 & 0.0314 & 0.0314 \\
0.9 & 0.4034 & 0.408 & 0.4081 \\
1 & 0.7854 & 0.7854 & 0.7854 \\
\hline
\end{tabular}


Table 4

Distribution of the component $q_{3} b /\left(k^{\text {skin }}\right)\left(x_{2}=1 / 2, z\right)$ of the heat flux along the thickness at the station $x_{2}=1 / 2$ for the sandwich considered in Fig. 6

\begin{tabular}{llll}
\hline$z / h$ & Exact & ESL_3_C & ESL_SC_C \\
\hline 0 & -0.0649 & -0.0638 & -0.0646 \\
0.1 & -0.0657 & -0.0661 & -0.0662 \\
$0.2-$ & -0.0681 & -0.0688 & -0.0684 \\
$0.2+$ & -0.0681 & -0.0688 & -0.0684 \\
0.3 & -0.3958 & -0.3684 & -0.3526 \\
0.4 & -0.7334 & -0.7032 & -0.6904 \\
0.5 & -1.0890 & -1.0733 & -1.0733 \\
0.6 & -1.4716 & -1.4786 & -1.4922 \\
0.7 & -1.8905 & -1.9191 & -1.9365 \\
$0.8-$ & -2.3562 & -2.3949 & -2.3953 \\
$0.8+$ & -2.3562 & -2.3949 & -2.3953 \\
0.9 & -2.3903 & -2.4000 & -2.3999 \\
1 & -2.4835 & -2.4054 & -2.4045 \\
\hline
\end{tabular}

Layer 2

$0.463644 T t \cosh (z)+0.242137 T t \sinh (z)$

The ESL_P3_C (cf. Eq. (8)) approximation gives: Layer 1

$$
\operatorname{Ttz}\left(0.120252 z^{2}+0.140765 z+0.790032\right)
$$

Layer 2

$0.120252 T t(z+1.42332)\left(z^{2}-0.252738 z+2.68433\right)$

The ESL_SC_C (cf. Eq. (8)) approximation gives:

Layer 1

$$
\begin{aligned}
& \operatorname{Tt}(1.11107 z+0.030123 \cos (\pi z) \\
& \quad-0.0823937 \sin (\pi z)-0.030123)
\end{aligned}
$$

Layer 2

$$
\begin{aligned}
& \operatorname{Tt}(0.602851 z+0.030123 \cos (\pi z) \\
& \quad-0.0823937 \sin (\pi z)+0.427272)
\end{aligned}
$$

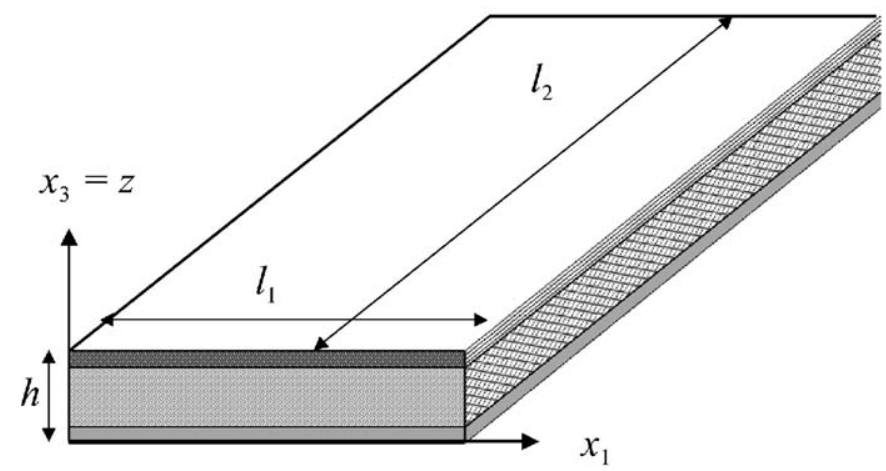

top skin material $m^{\text {skin_t }}$

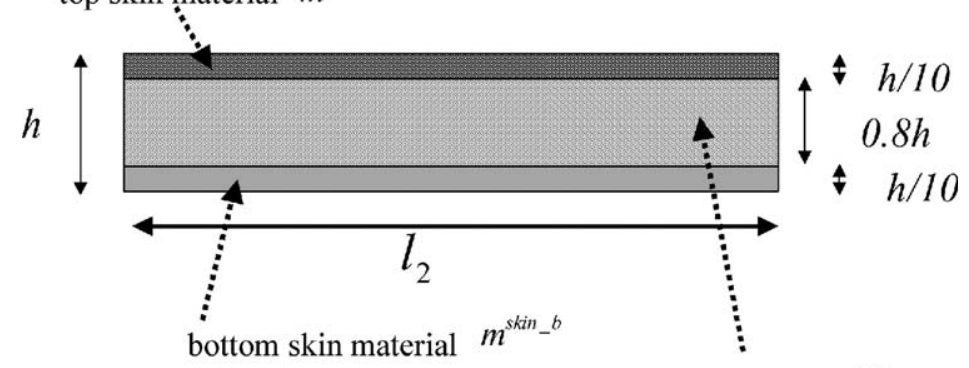

core material $m^{\text {core }}$

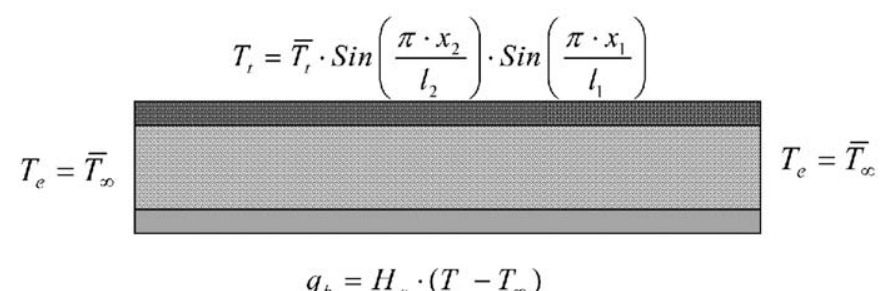

Fig. 7. Geometric properties, thermal loading and thermal boundary conditions for the three-dimensional sandwich problem. 
In Fig. 5, are compared the numerical results for ESL_3_C, ESL_SC_C, DL and CDL approximations with the exact solution, and a very good agreement occurs for all these models.

In this case the number of unknown functions is 2 for ESL_3_C, ESL_SC_C and CDL models.

\subsection{Computations of the heat conduction in a plane sandwich media}

By using both exact solutions provided by Eqs. (1)(4), the above refined models (ESL_3_C and ESL_SC_C), numerical calculations of temperature and heat flux are obtained for a rectangular $(l / b=2)$ symmetric sandwich media for which geometry and thermal properties, as well as boundary conditions are specified in Fig. 6. The sandwich is composed of an isotropic core of thickness $b-2 h$, and isotropic skins of equal thickness $h=0.2 b$, and is submitted to a simply sinusoidal temperature loading given by $\bar{T}^{\mathrm{t}}=T_{0} \sin \left(\pi x_{2} /\right.$ $l$ ) with $T_{0}=1$ at its top edge, while zero temperature is prescribed on the other three edges. Temperature distributions along the thickness of the sandwich are given in Table 2 at station $x_{2}=1 / 2$ of the cross-section of the sandwich for the exact solution $T_{\text {(exact) }}$, the distribution of the components $q_{\alpha}, \alpha=2$ or 3 , of the heat flux along the thickness of the sandwich media are compared with the exact calculations $q_{\alpha(\text { exact })}$ in Table 3 ( $q_{2}$ component at the station $\left.x_{2}=1\right)$ and in Table $4\left(q_{3}\right.$ component along the edge $x_{2}=l / 2$ ).

We use dimensionless quantities $z / h, T / T_{0} q b /\left(k^{\text {skin }}\right)$.

Of course, due to both in plane and the no time dependence character of this thermal problem, $x_{1}$ and $t$ have been disregarded. Results from the ESL 3 _C and ESL_SC_C are in very good agreement with the exact calculations.

\subsection{Computations of the heat conduction in a $3 D$ sandwich media}

We consider a parallelepipedic media corresponding to a laminated composite for which we use geometrical definition and thermal loading as defined in Fig. 7.

Physical properties are given as:
Table 5

Distribution of the temperature $\left(x_{1}=1 / 2, x_{2}=1 / 2, z\right)$ along the thickness at the centre of the plate for the sandwich media considered in Fig. 7

\begin{tabular}{llll}
\hline$z / h$ & Exact & ESL_3_C & ESL_SC_C \\
\hline 0.00 & 360.081 & 360.081 & 360.081 \\
0.05 & 360.082 & 360.082 & 360.082 \\
$0.10-$ & 360.084 & 360.084 & 360.084 \\
$0.10+$ & 360.084 & 360.084 & 360.084 \\
0.20 & 364.031 & 364.017 & 364.022 \\
0.30 & 367.980 & 367.954 & 367.964 \\
0.40 & 371.931 & 371.897 & 371.911 \\
0.50 & 375.882 & 375.845 & 375.866 \\
0.60 & 379.836 & 379.802 & 379.828 \\
0.70 & 383.791 & 383.769 & 383.797 \\
0.80 & 387.748 & 387.747 & 387.771 \\
$0.90-$ & 391.707 & 391.737 & 391.750 \\
$0.90+$ & 391.707 & 391.737 & 391.750 \\
0.95 & 392.341 & 392.367 & 392.375 \\
1.00 & 393.000 & 393.000 & 393.000 \\
\hline
\end{tabular}

Temperatures are given in $\mathrm{K}$.

Table 6

Distribution of the heat flux component $q_{1}\left(x_{1}=0, x_{2}=1 / 2, z\right)$ along the thickness of the plate for the sandwich media considered in Fig. 7

\begin{tabular}{lrrr}
\hline$z / h$ & \multicolumn{1}{l}{ Exact } & \multicolumn{1}{l}{ ESL_3_C } & ESL_SC_C \\
\hline 0.00 & $-34,561.435$ & $-34,561.507$ & $-34,561.547$ \\
0.05 & $-34,562.152$ & $-34,562.191$ & $-34,562.216$ \\
$0.10-$ & $-34,563.040$ & $-34,563.044$ & $-34,563.055$ \\
$0.10+$ & -33.088 & -33.088 & -33.088 \\
0.20 & -35.035 & -35.028 & -35.030 \\
0.30 & -36.982 & -36.970 & -36.974 \\
0.40 & -38.931 & -38.914 & -38.921 \\
0.50 & -40.880 & -40.862 & -40.872 \\
0.60 & -42.830 & -42.814 & -42.826 \\
0.70 & -44.781 & -44.770 & -44.784 \\
0.80 & -46.733 & -46.732 & -46.744 \\
$0.90-$ & -48.685 & -48.700 & -48.706 \\
$0.90+$ & -155.049 & -155.096 & -155.116 \\
0.95 & -156.045 & -156.085 & -156.097 \\
1.00 & -157.080 & -157.080 & -157.080
\end{tabular}

Heat flux values are given in $\mathrm{W} / \mathrm{m}^{2} \mathrm{~K}$.

Results are given for a thin square plate $\left(l_{1}=1 \mathrm{~m}\right.$; $h=0.01 \mathrm{~m}$ ), in Tables 5-8.

We give the results for ESL 3 _C, ESL_SC_C, in comparison with the exact solution.

It clearly appears that all the interfaces conditions are satisfied and, in general, for temperature and compo-

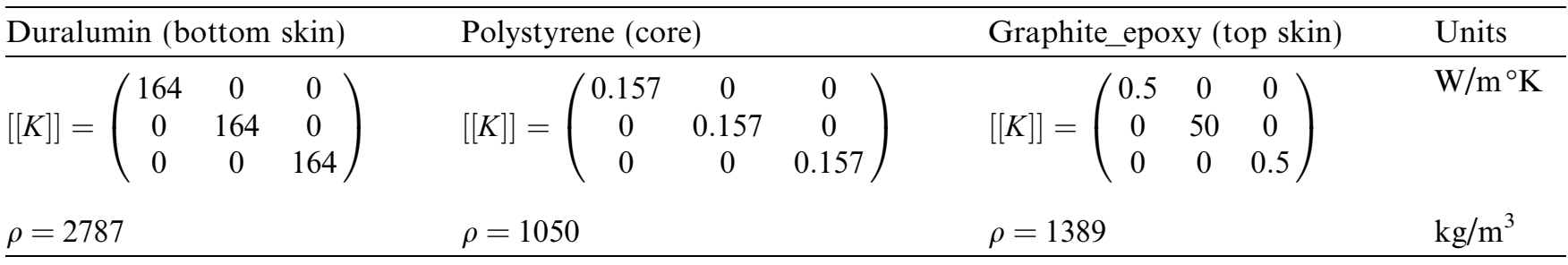


Table 7

Distribution of the heat flux component $q_{2}\left(x_{1}=1 / 2, x_{2}=0, z\right)$ along the thickness of the plate for the sandwich media considered in Fig. 7

\begin{tabular}{lrrr}
\hline$z / h$ & \multicolumn{1}{l}{ Exact } & \multicolumn{1}{c}{ ESL_3_C } & \multicolumn{1}{c}{ ESL_SC_C } \\
\hline 0.00 & $-34,561.435$ & $-34,561.507$ & $-34,561.547$ \\
0.05 & $-34,562.152$ & $-34,562.191$ & $-34,562.216$ \\
$0.10-$ & $-34,563.040$ & $-34,563.044$ & $-34,563.055$ \\
$0.10+$ & -33.088 & -33.088 & -33.088 \\
0.20 & -35.035 & -35.028 & -35.030 \\
0.30 & -36.982 & -36.970 & -36.974 \\
0.40 & -38.931 & -38.914 & -38.921 \\
0.50 & -40.880 & -40.862 & -40.872 \\
0.60 & -42.830 & -42.814 & -42.826 \\
0.70 & -44.781 & -44.770 & -44.784 \\
0.80 & -46.733 & -46.732 & -46.744 \\
$0.90-$ & -48.685 & -48.700 & -48.706 \\
$0.90+$ & $-15,504.889$ & $-15,509.638$ & $-15,511.563$ \\
0.95 & $-15,604.482$ & $-15,608.506$ & $-15,609.745$ \\
1.00 & $-15,707.963$ & $-15,707.963$ & $-15,707.963$ \\
\hline
\end{tabular}

Heat flux values are given in $\mathrm{W} / \mathrm{m}^{2} \mathrm{~K}$.

Table 8

Distribution of the heat flux component $q_{3}\left(x_{1}=1 / 2, x_{2}=1 / 2, z\right)$ along the thickness at the centre of the plate for the sandwich media considered in Fig. 7

\begin{tabular}{llll}
\hline$z / h$ & Exact & ESL_3_C & ESL_SC_C \\
\hline 0.00 & -402.485 & -402.485 & -402.486 \\
0.05 & -511.064 & -478.717 & -464.542 \\
$0.10-$ & -619.645 & -617.351 & -617.963 \\
$0.10+$ & -619.645 & -617.351 & -617.963 \\
0.20 & -619.859 & -617.796 & -618.503 \\
0.30 & -620.085 & -618.479 & -619.323 \\
0.40 & -620.324 & -619.402 & -620.343 \\
0.50 & -620.575 & -620.563 & -621.464 \\
0.60 & -620.838 & -621.964 & -622.575 \\
0.70 & -621.113 & -623.603 & -623.568 \\
0.80 & -621.400 & -625.482 & -624.346 \\
$0.90-$ & -621.700 & -627.599 & -624.833 \\
$0.90+$ & -621.700 & -627.599 & -624.833 \\
0.95 & -646.377 & -631.256 & -625.208 \\
1.00 & -671.215 & -635.103 & -625.302 \\
\hline
\end{tabular}

Heat flux values are given in $\mathrm{W} / \mathrm{m}^{2} \mathrm{~K}$.

nents of heat flux, results are in good agreement with exact calculations for this three-dimensional problem.

\section{Summary and conclusions}

In this paper, a new simple refined computational model is proposed to analyze heat conduction in composite laminates. This model based on an equivalent single layer approach allows to satisfy the compatibility conditions for temperatures and the heat flux at layer interfaces, as well as the boundary conditions. Numerical comparisons made between exact solutions, equivalent single layer with polynomial or sinusoidal surimposed functions to the classic linear distribution, shows the efficiency of the approach, in various cases: laminated three-dimensional problems, interface discontinuity by incorporating thermal resistance, as well as boundary conditions combining temperature and heat flux (conduction and convection) requirements. It will be easy to derive a new finite element from this formulation, having just to interpolate the independent generalized temperatures. Thus, choosing either the ESL_3_C or ESL_SC_C models, only two generalized temperatures have to be interpolated, the continuity requirement being $\mathrm{C}^{0}$.

This paper showed the efficiency of the Heaviside step function to assure automatically the interface continuity for temperatures, and those for the heat flux by eliminating the additional unknowns by satisfying both interface continuity and boundary conditions on the heat flux.

\section{References}

[1] Savoia M, Reddy JN. Three-dimensional thermal analysis of laminated composite plates. Int J Solids Struct 1995;32(5): 593-608.

[2] Padovan J. Solution of transient temperature fields in laminated anisotropic slabs and cylinders. Int J Eng Sci 1975;13: 247-60.

[3] Padovan J. Thermoelasticity of anisotropic generally laminated slabs subject to spacially periodic thermal loads. ASME J Appl Mech 1975(June):341-6.

[4] Mikhailov MD, Ozisik NL, Vulchanov NL. Diffusion in composite layers with automatic solution of the eigenvalue problem. Int J Heat Mass Transfer 1983;26(8):1131-41.

[5] Han Lit S. Periodic heat conduction through composite panels. AIAA J Thermophys 1986;1(2):184-6.

[6] Maewal A, Bache TC, Hegemier GA. A continuum model for diffusion in laminated composite media. J Heat Transfer 1976:133-8.

[7] Bose A, Surana KS. Axisymmetric shell element for heat conduction in laminated composites. Comput Struct 1993;48(1): 33-49.

[8] Chattopadhyay A, Li J, Gu H. Coupled thermo-piezoelectricmechanical model for smart composite laminates. AIAA J 1999;37(12):1633-8.

[9] Carrera E. Theories for thermal stress analysis of orthotropic multilayered plates. J Thermal Stresses 2000;23:797-831.

[10] Zhou X, Chattopadhyay A, Gu H. Dynamic responses of smart composites using a coupled thermo-piezoelectric-mechanical model. AIAA J 2000;38(10):1939-48.

[11] Surana KS, Orth NJ. Elements for heat conduction in laminated composites. Comput Struct 1989;33(5):1251-65.

[12] Surana KS, Abulsaleh G. Curved shell elements for heat conduction with p-approximation in the shell thickness direction. Comput Struct 1990;34(6):861-80.

[13] Surana KS, Orth NJ. p-Version hierarchical three-dimensional curved shell element for heat conduction. Comput Mech 1991;7:341-53.

[14] Bose A, Surana KS. Piecewise hierarchical p-version curved shell finite element for heat conduction in laminated composites. Comput Struct 1993;49(2):283-300.

[15] Carrera E. Temperature profile influence on layered plates response considering classical and advanced theories. AIAA J 2002;40(9):1885-96. 
[16] Touratier M. Elastic wave propagation in rectangular rods reinforced by unidirectional fiber composites. Int J Eng Sci 1980;18:931-46.

[17] Touratier M. A refined theory of laminated shallow shells. Int $\mathbf{J}$ Solids Struct 1992;29(11):1401-15.

[18] Carslaw HS, Jaeger JC. Conduction of heat in solids. Oxford Science Publications; 1959.
[19] Ossadzow-David C, Touratier M. Multilayered piezoelectric refined plate theory. AIAA J 2003;41(1):90-9.

[20] Wolfram S. The mathematica book, mathematica version 4. 4th ed. Cambridge University Press; 1999.

[21] Blanc M, Touratier M. A constrained discrete layer model for heat conduction in laminated composites. Comput Struct 2005;83:1705-18. 